Editor's Note: These short critical reviews of recent papers in the Journal, written exclusively by graduate students or postdoctoral fellows, are intended to summarize the important findings of the paper and provide additional insight and comentary. For more information on the format and purpose of the Journal Club, please see http://www.jneurosci.org/misc/ifa_features.shtml.

\title{
Anatomy of the Corpus Callosum Reveals Its Function
}

\author{
Eric Mooshagian \\ Department of Psychology, University of California, Los Angeles, California 90095-1563 \\ Review of Wahl et al. (http://www.jneurosci.org/cgi/content/full/27/45/12132)
}

The corpus callosum (CC) comprises axons connecting the cortices of the two cerebral hemispheres and is the principal white matter fiber bundle in the brain. As recently as the mid 20th century, the CC was thought to serve no other purpose than preventing the two hemispheres from collapsing on one another (Bogen, 1979). This cynical view was attributable to the failure of the Van Wagenen/Akelaitis split-brain surgery to reveal strong disconnection symptoms. The work of Myers and Sperry (1958) in the 1950s changed this by definitively confirming the functional role of the CC in the interhemispheric transfer of visual information in animals. Since then, the structure and function of the CC have remained topics of continuous investigation (Zaidel and Iacoboni, 2003). In particular, researchers have asked how callosal structure relates to callosal function (i.e., hemispheric specialization and interaction). One pioneering study of callosal morphology relied on light microscopy of postmortem specimens to look at callosal structure and fiber composition (Aboitiz et al., 1992), but most anatomical studies of humans have relied on structural magnetic resonance imaging (MRI) morphometry of midsagittal cross-section

Received Dec. 7, 2007; revised Jan. 7, 2008; accepted Jan. 10, 2008.

I thank Teresa Esch and Eran Zaidel and the members of his laboratory for constructive comments and discussion on a previous draft of this manuscript.

Correspondence should be addressed to Eric Mooshagian, Department of Psychology, University of California, Los Angeles, Box 951563, Los Angeles, CA 90095-1563. E-mail: emooshag@ucla.edu.

DOI:10.1523/JNEUROSCI.5426-07.2008

Copyright $\odot$ 2008 Society for Neuroscience $\quad$ 0270-6474/08/281535-02\$15.00/0 views of the CC. In contrast, a few recent studies have used diffusion tensor imaging (DTI) methods to re-evaluate callosal topography (for discussion, see Wahl et al., 2007). These methods challenge the conventional partitioning schemes used to divide the CC into functionally significant regions (Witelson, 1989).

In their recent article published in The Journal of Neuroscience, Wahl et al. (2007) revisit the issue of CC topography using functional MRI (fMRI) and DTI to assess callosal motor fiber (CMF) microstructure in vivo. Their participants performed a simple, visually guided motor task that included alternating blocks of pursing lips, flexing left-hand fingers, right-hand fingers, left toes, and right toes, and a rest condition, all while being scanned. DTI analysis allows one to quantify the magnitude and direction of water diffusion (i.e., fractional anisotropy). Because diffusion of water molecules is hindered by the axonal membrane and myelin sheath, the molecules disperse primarily in the direction of the fiber tract, thus allowing one to infer the overall orientation of white matter fibers.

The results of this study revealed a topographic organization of CMFs in the CC. Whereas previous anatomical studies suggested that CMFs cross primarily through the anterior midbody of the CC, Wahl et al. (2007) found that CMFs cross through the posterior body and isthmus [Wahl et al. (2007), their Fig. $2 b$ (http:// www.jneurosci.org/cgi/content/full/27/ $45 / 12132 / \mathrm{F} 2)]$. These results are in agreement with other previous DTI studies ex- amining callosal topography, suggesting a more posterior crossing of CMFs (for discussion, see Wahl et al., 2007). In addition, the present study goes beyond a demonstration of topography by revealing, for the first time, a clear somatotopy of CMFs; hand fibers were situated ventral and anterior to foot fibers, and lip fibers, for the one subject in which they were visualized successfully, were localized anterior to the hand fibers [Wahl et al. (2007), their Figs. $2 b$ (http://www.jneurosci.org/ cgi/content/full/27/45/12132/F2), 4 (http:// www.jneurosci.org/cgi/content/full/27/45/ 12132/F4)].

Next, the authors extended this imaging approach using a paired-pulse transcranial magnetic stimulation (TMS) paradigm to assess the functional connectivity between the primary (M1) hand areas of each cerebral hemisphere, thereby measuring interhemispheric inhibition. The paired-pulse TMS approach involves measuring the effect of a conditioning pulse on the motor-evoked potential (MEP) elicited by a second, test pulse. The conditioning pulse was given over the left $\mathrm{M} 1$, and the test pulse was given over the right M1. The intensity of the conditioning pulse varied between 100 and $150 \%$ of individuals' resting motor threshold, in $10 \%$ intervals. Interhemispheric inhibition threshold was measured as the percentage of maximum stimulator output required for a 25 or $50 \%$ inhibition. The authors found a positive correlation between fractional anisotropy of hand CMFs and the magnitude of interhemispheric inhibition measured physiologi- 
cally [Wahl et al. (2007), their Fig. 5a (http://www.jneurosci.org/cgi/content/ full/27/45/12132/F5)] and a negative correlation between fractional anisotropy of hand CMFs with threshold intensity [Wahl et al. (2007), their Fig. 5b (http:// www.jneurosci.org/cgi/content/full/27/ 45/12132/F5)]. Fractional anisotropy of foot CMFs did not correlate with either the magnitude or the threshold intensity of interhemispheric inhibition [Wahl et al. (2007), their Fig. 5c,d (http://www. jneurosci.org/cgi/content/full/27/45/ $12132 / F 5)]$. In short, the results demonstrate that the microstructure (measured by fractional anisotropy) of callosal fibers predicts their function (measured by interhemispheric inhibition).

Heretofore, the relationship between CC morphometry and hemispheric specialization has been explored by correlating the midsagittal callosal area with performance on behavioral laterality tests (Clarke and Zaidel, 1994). These studies have relied on partitioning schemes that obtain regions based on fractions of the longest rostral-caudal CC length (Witelson, 1989). Thus, researchers inferred the function of a callosal region and, in turn, the fibers within the region, from behavior (Clarke and Zaidel, 1994). In their study, Wahl et al. (2007) used DTI to visualize, on an individual basis, where CMFs cross in the CC. The authors suggest that their approach may provide a useful way to better understand the functional relevance of fiber pathways in the human brain. Indeed, the combined use of fractional anisotropy with TMS, particularly the paired-pulse design, offers a powerful new approach to examining the link between structure and function of callosal fibers. Moreover, it is now possible to go past gross morphometry across subjects and take into account differences in individual anatomy, which show significant variation [Wahl et al. (2007), their Fig. 3 (http://www.jneurosci.org/cgi/content/full/27/45/12132/F3)]. Specifically, data of the sort presented by Wahl et al. (2007) allow one to partition the CC into functionally meaningful regions. Moreover, the promise of the techniques described in their study is that they can relate individual variations in brain structure to individual differences in behavior.

The paired-pulse TMS approach to linking the observed microstructure to function is not without its limitations, however; it seems best suited to studying CMFs, as it was used in this study. It is not clear how TMS can be easily implemented to demonstrate connectivity for fibers subserving other functions (e.g., auditory or visual). For example, the phosphenes evoked by TMS over visual cortical regions are subjective, and they do not provide a convenient, quantifiable measure as do MEPs for TMS over motor regions.

The work by Wahl et al. (2007) leaves a few open questions. For example, they only look at homotopic cortical regions; their DTI analysis was constrained such that heterotopic connections were not considered. It would also be useful to apply the same DTI analysis approach to visualization of heterotopic callosal fibers (e.g., those between premotor or supplementary motor and primary motor cortices) and determine whether these fibers share the same somatotopy observed in their study. Furthermore, Wahl et al. (2007) only considered left-to-right interhemispheric inhibition, although asymmetry in callosal transfer has been documented (Zaidel and Iacoboni, 2003). Thus, it remains to be seen how CC topog- raphy and somatotopy relate to asymmetrical callosal transfer.

In summary, Wahl et al. (2007) demonstrate that novel MRI and TMS techniques can be effectively combined to investigate the link between structure and function in vivo in human subjects. Their results suggest that the role of the $\mathrm{CC}$ in interhemispheric integration, as it relates to structure, should be re-examined in light of the new approaches to studying the morphometry and morphology of the CC. It will be useful in future experiments to reconsider the relationship between individual callosal anatomy and behavioral laterality measures (Clarke and Zaidel, 1994).

\section{References}

Aboitiz F, Scheibel AB, Fisher RS, Zaidel E (1992) Fiber composition of the human corpus callosum. Brain Res 598:143-153.

Bogen JE (1979) The callosal syndromes. In: Clinical neuropsychology (Heilman KM, Valenstein E, eds), pp 308-359. New York: Oxford UP.

Clarke JM, Zaidel E (1994) Anatomicalbehavioral relationships: corpus callosum morphometry and hemispheric specialization. Behav Brain Res 64:185-202.

Myers RE, Sperry RW (1958) Interhemispheric communication through the corpus callosum: mnemonic carry-over between the hemispheres. AMA Arch Neurol Psychiatry 80:298-303.

Wahl M, Lauterbach-Soon B, Hattingen E, Jung P, Singer O, Volz S, Klein JC, Steinmetz H, Ziemann U (2007) Human motor corpus callosum: topography, somatotopy, and link between microstructure and function. J Neurosci 27:12132-12138.

Witelson SF (1989) Hand and sex differences in the isthmus and genu of the human corpus callosum. A postmortem morphological study. Brain 112:799-835.

Zaidel E, Iacoboni M, eds (2003) The parallel brain: the cognitive neuroscience of the corpus callosum. Cambridge, MA: MIT. 